\title{
Síndroma do túnel cárpico ulcero-mutilante: relato de um
} caso

Inês Torrinha Leão, ${ }^{1}$ Daniel Kiessling, ${ }^{1}$ Fabíola Ferreira ${ }^{2}$

\section{RESUMO}

Introdução: A síndroma do túnel cárpico (STC) é a mononeuropatia focal compressiva mais comum observada na prática clínica. Em alguns casos, a apresentação clínica pode ser atípica e, em estadios avançados, pode ocorrer uma variedade de manifestações cutâneas.

Descrição do caso: Doente do sexo masculino, com 84 anos, pertencente a uma família alargada de classe social média, com antecedentes de hipertensão arterial, dislipidemia, neoplasia da próstata e cirurgia bilateral a STC. O doente foi observado na sua unidade de saúde familiar por avulsão parcial da unha no terceiro dedo da mão esquerda, em contexto de lesão em placa erosiva, sangrante e indolor, aparentemente não traumática no leito ungueal, com três meses de evolução. Pela suspeita de melanoma, o doente foi referenciado à consulta de dermatologia, mas entretanto, por apresentação de sinais inflamatórios recorrentes, foi submetido a vários ciclos de antibioterapia e desbridamento cirúrgico. Posteriormente, já em consulta de dermatologia, foi realizada biópsia que excluiu a suspeita diagnóstica de neoplasia e foi colocada a hipótese de STC ulcero-mutilante, confirmada por eletromiografia e pela clínica sugestiva.

Comentário: A STC ulcero-mutilante é uma variante rara de uma patologia muito frequente nos cuidados de saúde primários. Assim, o médico de família deve estar alerta para a sua existência, iniciando a abordagem diagnóstica, assegurando a necessária articulação com as diferentes valências hospitalares, a continuidade de cuidados e a prevenção de complicações.

Palavras-chave: Síndroma de túnel cárpico; Acro-osteólise; Úlcera cutânea.

\section{INTRODUÇÃO}

A síndroma do túnel cárpico (STC) é a mononeuropatia focal compressiva mais comum observada na prática clínica. ${ }^{1}$ Refere-se ao conjunto de sinais e sintomas provocados pela compressão do nervo mediano à medida que atravessa o túnel cárpico. ${ }^{2-3}$

Os doentes geralmente apresentam uma tríade clássica de sintomas, que incluem dor noturna, parestesias e atrofia tenar. ${ }^{3}$ Habitualmente estas manifestações envolvem os primeiros três dedos da mão devido ao território de inervação do nervo mediano. ${ }^{2-3}$

Em alguns casos, a apresentação clínica pode ser atípica e, em estadios avançados, como o STC ulcero-mutilante, pode ocorrer uma variedade de manifestações

1. Médico Interno de Medicina Geral e Familiar. USF Vale do Vez, ULS do Alto Minho. 2. Médica Assistente Graduada de Medicina Geral e Familiar. USF Vale do Vez, ULS do Alto Minho. cutâneas, como o eritema, edema, esclerodactilia, atrofia cutânea e cianose, lesões bolhosas, úlceras e alterações das unhas. ${ }^{1,4}$

\section{DESCRIÇÃO DO CASO CLÍNICO}

Doente do sexo masculino, 84 anos de idade, raça caucasiana, viúvo, independente para as atividades de vida diária, reformado, previamente trabalhador agrícola. Insere-se numa família alargada, altamente funcional e pertencente à classe média de Graffar. Apresenta como antecedentes pessoais: hipertensão arterial diagnosticada em 2012, medicada com perindopril + indapamida 4+1,25mg um comprimido de manhã; dislipidemia mista diagnosticada em 2012, medicada com fenofibrato $145 \mathrm{mg}$ e sinvastatina $20 \mathrm{mg}$ um comprimido ao jantar; e adenocarcinoma da próstata diagnosticada em 2012, tratada com hormonoterapia, mantendo acompanhamento hospitalar. 


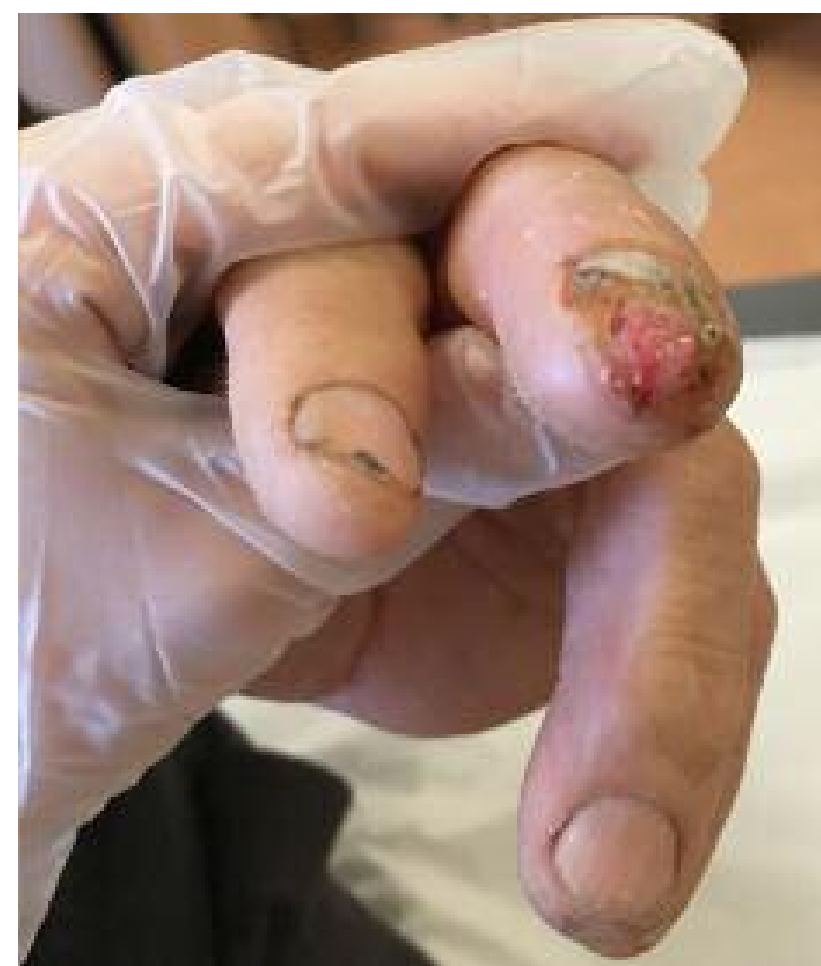

Figura 1. Primeira consulta: lesão em placa erosiva sangrante e indolor no terceiro dedo da mão esquerda.

Foi submetido a correção cirúrgica de STC bilateral, à direita em 2008 e à esquerda em 2010. Após a última cirurgia manteve parestesias e hipoalgesia dos segundo e terceiro dedos da mão esquerda, com melhoria da força muscular. O doente não valorizou os sintomas, uma vez que não interferiam na realização das suas atividades diárias. Sem antecedentes familiares relevantes.

\section{História da doença atual}

O doente recorreu à consulta aberta na sua unidade de saúde familiar (USF) por avulsão parcial da unha do terceiro dedo da mão esquerda, quinze dias antes da consulta, devido à existência de uma lesão não traumática presente no leito ungueal. Essa lesão teria três meses de evolução e, de acordo com o doente, previamente no seu lugar teria um nevo. Ao exame objetivo apresentava lesão em placa erosiva, sangrante e indolor, localizada na polpa do terceiro dedo da mão esquerda (Figura 1).

Pela suspeita de melanoma, o doente foi referenciado à consulta de dermatologia, mantendo cuidados de penso na USF com betadine ${ }^{\circledR}$.

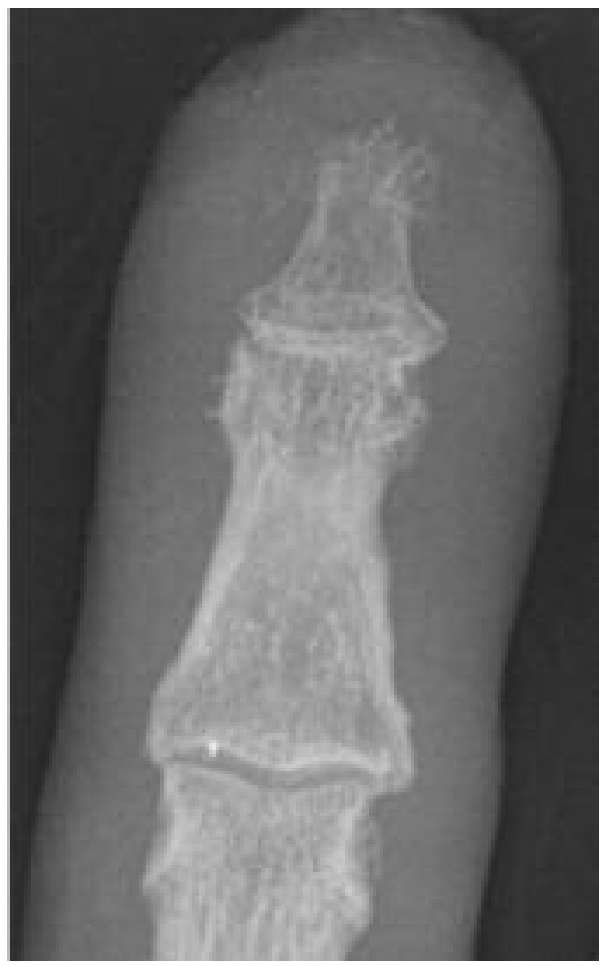

Figura 2. Primeira consulta: radiografia do terceiro dedo da mão esquerda - acro-osteólise no tofo da falange distal.

Entretanto, cerca de um mês depois, estando ainda a aguardar consulta de dermatologia, regressou à consulta aberta da USF pelo aparecimento de sinais inflamatórios no dedo afetado compatíveis com quadro de dactilite, ainda que sem queixas álgicas associadas. Realizou radiografia dos dedos da mão (Figura 2), que mostrou acro-osteólise no tofo da falange distal do terceiro dedo da mão esquerda, tendo sido encaminhado para o serviço de urgência (SU) de ortopedia, onde foi levantada a hipótese de osteomielite, realizado desbridamento cirúrgico e instituída antibioterapia com levofloxacina 500mg um comprimido de 12 em 12 horas e metronidazol 250mg dois comprimidos a cada oito horas durante 10 dias e dada indicação para continuação de cuidados de penso, com betadine ${ }^{\circledR}$, na sua USF (Figuras 3 e 4).

Três dias mais tarde, já em consulta de dermatologia, foi realizada biópsia pela suspeita de melanoma, mas esta apenas revelou aspetos remanescentes de tecido de granulação/granuloma piogénico incipiente.

O doente voltou à consulta de dermatologia cerca de uma semana mais tarde, realizou nova radiografia a 


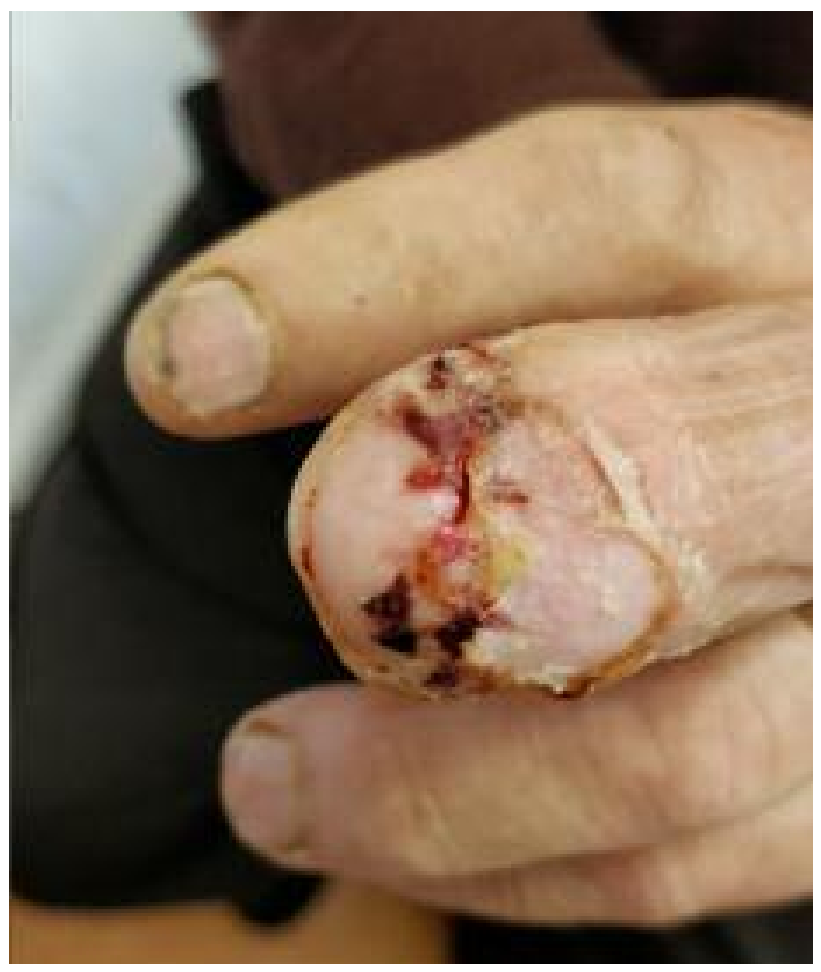

Figura 3. Segunda consulta: ferida pós desbridamento cirúrgico e início de antibioterapia.

nível hospitalar pela ausência de melhoria do quadro. Face aos achados radiográficos e clínicos (Figura 5) optou-se por repetir biópsia e reinstituir antibioterapia com cefaclor 500mg um comprimido de 12 em 12 horas durante 10 dias.

Cerca de oito dias após última consulta foi novamente observado por dermatologia. Os resultados anatomopatológicos da segunda biópsia excluíram mais uma vez lesão tumoral. Devido aos antecedentes de STC e à ausência de sensibilidade dolorosa e tátil do doente durante todo o quadro foi levantada a hipótese de STC ulcero-mutilante e solicitada colaboração de ortopedia.

Enquanto aguardava a consulta de ortopedia foi solicitada eletromiografia dos membros superiores, pelo seu médico de família, que confirmou STC esquerdo de grau severo.

Em consulta, a ortopedia ponderou a amputação pela persistência dos sinais inflamatórios apesar dos vários ciclos de antibioterapia e pela possibilidade de recorrência de novas lesões cutâneas. No entanto, após

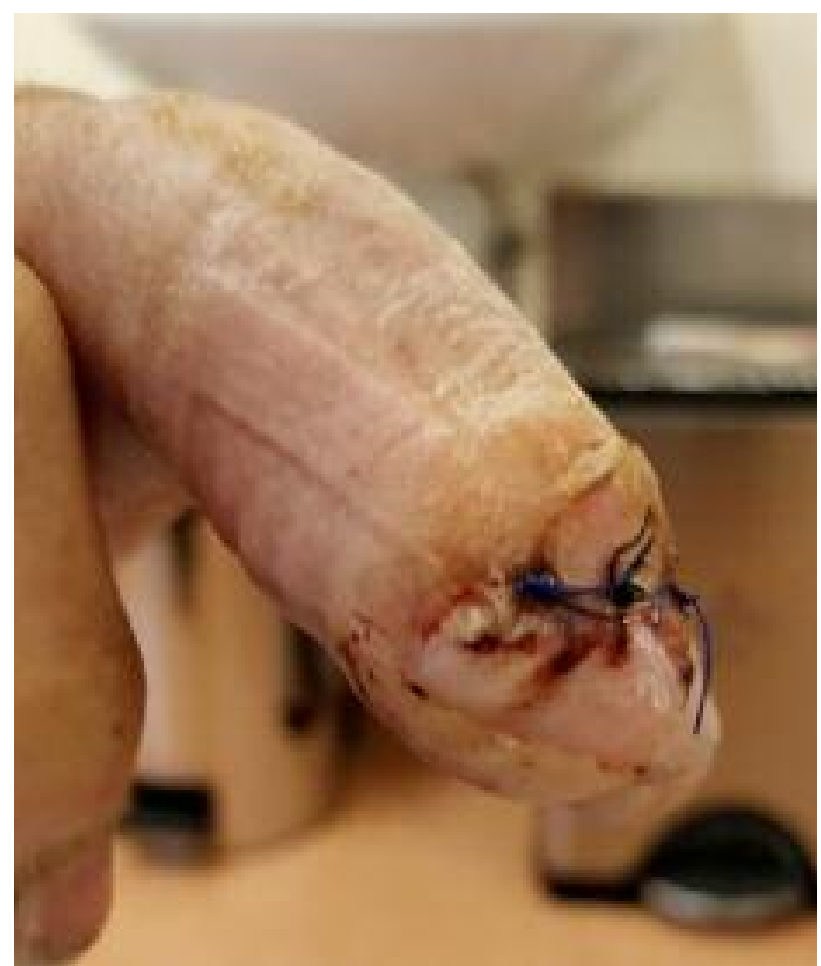

Figura 4. Segunda consulta: ferida após desbridamento cirúrgico e início de antibioterapia, em perfil.

último ciclo de antibioterapia com flucloxacilina $500 \mathrm{~g}$ um comprimido de oito em oito horas durante 14 dias, os sinais inflamatórios acabaram por reverter (Figura 7), tendo-se optado de forma partilhada por manter uma atitude expectante. Atualmente mantém hipostesia dolorosa e tátil e ligeira limitação da flexão das falanges média e distal. Sem novas lesões cutâneas desde então.

\section{COMENTÁRIO}

As úlceras cutâneas associadas à STC são raras, tendo sido inicialmente descritas pela literatura científica em 1979. ${ }^{5}$ Desde então poucos casos têm sido publicados, estimando-se que o envolvimento cutâneo na STC grave seja de $20 \%{ }^{6}$

Em contraste com a alta prevalência de STC em pessoas do sexo feminino quando comparadas com o sexo masculino (rácio de 4:1), ${ }^{7}$ na variante cutânea da STC foi demonstrada uma diminuição significativa deste rácio $(2: 1) .^{5}$

A perda de sensibilidade cutânea causada pela compressão autonómica das fibras do nervo mediano 


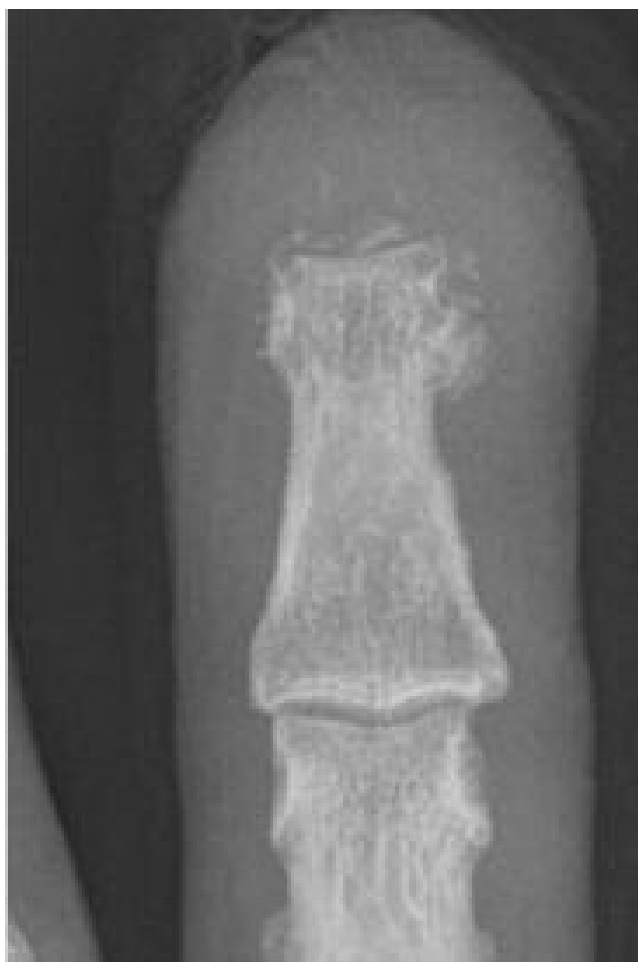

Figura 5. Consulta de dermatologia: radiografia do terceiro dedo da mão esquerda - avulsão da falange distal, acro-osteólise da cabeça da falange média.

predispõe ao aparecimento de úlceras cutâneas. Um dos fatores predisponentes para esta variante da STC é o trauma mecânico associado a trabalhos manuais..$^{2-3,8}$ A variante cutânea da STC é caracterizada pelo aparecimento de úlceras, bolhas e atrofia localizadas nas falanges distais dos primeiros três dedos da mão, mesmo sem a presença de sintomas neurológicos. As unhas podem apresentar onicodistrofia, onicólise e cutículas hiperqueratósicas. ${ }^{4}$ Em casos mais graves pode ocorrer envolvimento ósseo com acro-osteólise das falanges distais devido a oclusão vascular. ${ }^{3,9-10}$ Estes fatores predispõem a infeções bacterianas e, eventualmente, a mutilação das falanges.

Apesar de ser uma entidade rara, os médicos devem considerar a variante cutânea da STC quando se encontram na presença de lesões necróticas e bolhosas dos primeiros três dedos das mãos, especialmente quando existem sinais neurológicos.

O principal diagnóstico diferencial das lesões ulcerativas é a esclerose sistémica, mas que pode ser

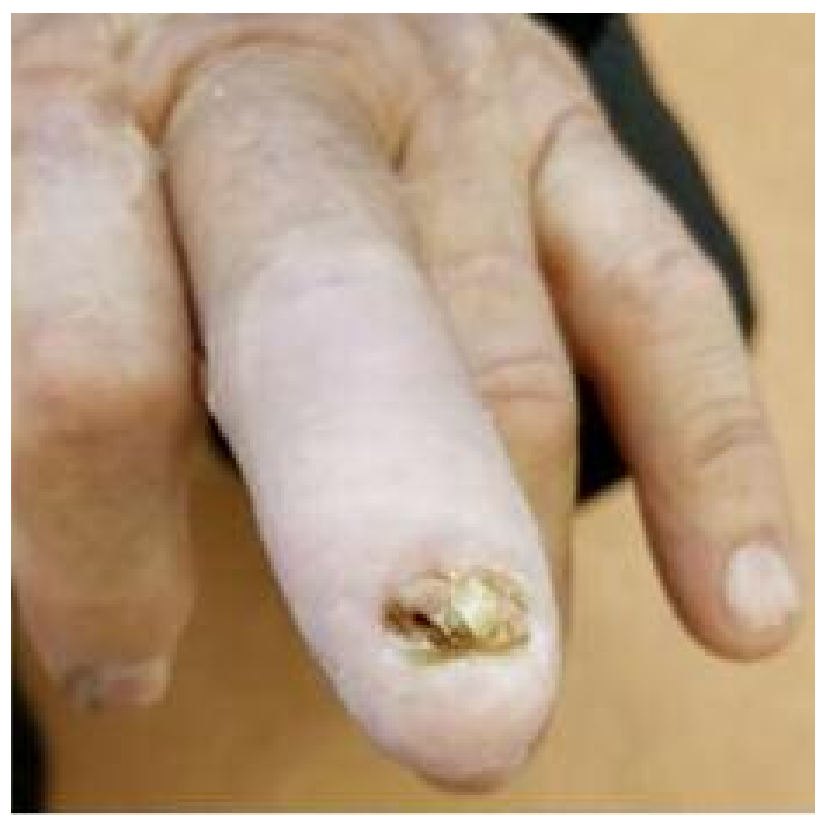

Figura 6. Consulta de reavaliação: terceiro dedo da mão esquerda após novo ciclo de antibioterapia com flucloxacilina.

excluída pela ausência de endurecimento da pele, assim como de alterações capilares. As lesões bolhosas devem ser diferenciadas do impetigo, pênfigo, herpes simplex ou herpes zoster. Nestes casos, as características clínicas da STC, como a hipostesia neste caso, aliadas à eletromiografia, permitem realizar o diagnóstico correto. A radiografia óssea é útil no sentido em que pode demonstrar a existência de acro-osteólise, que é usualmente resultado da deficiente vascularização distal, no entanto, não é essencial ao diagnóstico. ${ }^{10}$

Por se tratar de um diagnóstico raro, é muitas vezes necessária a exclusão de outros diagnósticos mais frequentes. Neste caso, pela descrição por parte do doente da existência de um nevo prévio, a biópsia foi importante para a exclusão de neoplasia.

A cirurgia de libertação do túnel cárpico é o tratamento de escolha e na maioria dos casos leva à cura. No caso descrito não se optou por este tratamento, pelo facto de o doente já ter sido submetido a cirurgia prévia, sem melhoria, e considerando também a vontade do mesmo.

A descrição deste caso clínico, que expõe uma variante rara, potencialmente mutilante, de uma patologia muito frequente nos cuidados de saúde primários, 
pretendeu alertar para a sua existência, assim como descrever a abordagem diagnóstica e terapêutica destes casos. O médico de família desempenhou, neste caso, um papel essencial na articulação dos cuidados, assim como na continuidade dos cuidados prestados ao doente e no aconselhamento para a prevenção de complicações, como o cuidado em relação a traumatismos e ao aparecimento de novas lesões.

\section{AGRADECIMENTOS}

À Dra Vera Teixeira, Assistente de Dermatologia da Unidade Local de Saúde do Alto Minho pelo apoio prestado na elaboração do presente artigo.

\section{REFERÊNCIAS BIBLIOGRÁFICAS}

1. Khan SK, Saeed I. Acral osteolysis in bilateral carpal tunnel syndrome. J Radiol Case Rep. 2008;2(2):3-4.

2. Fritz TM, Burg G, Böni R. Carpal tunnel syndrome with ulcer skin lesions. Dermatology. 2000;201(2):165-7.

3. Natale M, Spennato P, Bocchetti A, Fratta M, Savarese L, Rotondo M. Ulcerative and mutilating variant of carpal tunnel syndrome. Acta Neurochir. 2005;147(8):905-8.

4. Pereira N, Canelas MM, Vieira R, Figueiredo A. Síndrome do túnel cárpico: variante ulcero-mutilante [Carpal tunnel syndrome: ulcerative and mutilating variant]. Rev Soc Port Dermatol Venereol. 2011;69(3): 467-70. Portuguese

5. Bouvier M, Lejeune B, Rouillat M, Marionnet J. Les formes ulcéro-mutilantes du syndrome du canal carpien. Rev Rhum. 1979;46:169-76.
6. Aratari E, Regesta G, Rebora A. Carpal tunnel syndrome appearing with prominent skin symptoms. Arch Dermatol. 1984;120(4):517-9.

7. Tosti A, Morelli R, D'Alessandro R, Bassi F. Carpal tunnel syndrome presenting with ischemic skin lesions, acroosteolysis and nail changes. J Am Acad Dermatol. 1993;29(2 Pt 2):287-90.

8. Romaní J, Puig L, de Miguel G, de Moragas J.M. Carpal tunnel syndrome presenting as sclerodactylia, nail dystrophy and acro-osteolysis in a 60year-old woman. Dermatology. 1997;195(2):159-61.

9. Foti C, Romita P, Vestita M. Unusual presentation of carpal tunnel syndrome with cutaneous signs: a case report and review of the literature. Immunopharmacol Immunotoxicol. 2011;33(4):751-3.

10. Perdan-Pirkmajer K, Praprotnik S, Tomšic M. Digital ulcers as the first manifestation of carpal tunnel syndrome. Rheumatol Int. 2011;31(5): 685-6.

11. Cox NH, Large DM, Paterson WD, Ive FA. Blisters, ulceration and autonomic neuropathy in carpal tunnel syndrome. Br J Dermatol. 1992; 126(6):611-3.

\section{CONFLITO DE INTERESSES}

Os autores declaram não ter quaisquer conflitos de interesse.

\section{ENDEREÇO PARA CORRESPONDÊNCIA}

Inês Torrinha Leão

E-mail: ines.torrinha@gmail.com

https://orcid.org/0000-0002-5788-2433

Recebido em 18-12-2018

Aceite para publicação em 02-07-2019

\section{ABSTRACT}

\section{THE ULCERATIVE AND MUTILATING VARIANT OF CARPAL TUNNEL SYNDROME: A CASE REPORT}

Introduction: Carpal tunnel syndrome (CTS) is the most common compressive focal mononeuropathy in clinical practice. In some cases, the clinical presentation may be atypical and in advanced stages, a variety of cutaneous manifestations may occur.

Case summary: 84-year-old male patient from an extended middle-class family with a history of hypertension, dyslipidemia, prostate cancer, and bilateral CTS surgery. The patient was observed by his family physician because of partial avulsion of the nail on the third finger of the left hand. He had previously an erosive, painless, apparently non-traumatic, bleeding lesion on the nail bed of that finger with three months of evolution. Considering the suspicion of melanoma, the patient was referred to a dermatology specialist. Meanwhile, due to the presence of recurrent inflammatory signs, he underwent several cycles of antibiotic therapy and surgical debridement. Subsequently, in consultation with dermatology, the biopsy performed excluded the diagnostic suspicion of neoplasia, and the hypothesis of an ulcer-mutilating STC was placed and confirmed by electromyography and suggestive clinic.

Commentary: Ulcerative and mutilating CTS are a rare variant of a very common disease in Primary Care. Thus, the family physician should be alert to its existence, initiating the diagnostic approach, ensuring the necessary articulation with the different hospital specialties, and the continuity of care and prevention of complications.

Keywords: Carpal tunnel syndrome; Acro-osteolysis; Skin ulcer. 